TRANSACTIONS OF THE

AMERICAN MATHEMATICAL SOCIETY

Volume 196, 1974

\title{
THE SLIMMEST GEOMETRIC LATTICES
}

\author{
BY
}

THOMAS A. DOWLING( ${ }^{1}$ ) AND RICHARD M. WILSON(2)

ABSTRACT. The Whitney numbers of a finite geometric lattice $L$ of rank $r$ are the numbers $W_{k}$ of elements of rank $k$ and the coefficients $w_{k}$ of the characteristic polynomi al of $L$, for $0 \leq k \leq r$. We establish the following lower bounds for the $W_{k}$ and the absolute values $w_{k}^{+}=(-1)^{k} w_{k}$ and describe the lattices for which equality holds (nontrivially) in each case:

$$
W_{k} \geq\left(\begin{array}{l}
r-2 \\
k-1
\end{array}\right)(n-r)+\left(\begin{array}{l}
r \\
k
\end{array}\right), \quad w_{k}^{+} \geq\left(\begin{array}{l}
r-1 \\
k-1
\end{array}\right)(n-r)+\left(\begin{array}{l}
r \\
k
\end{array}\right),
$$

where $n=W_{1}$ is the number of points of $L$.

1. Introduction. Let $L$ be a finite geometric lattice of rank $r$. The numbers $W_{1}, W_{2}, W_{3}, \cdots, W_{r-1}$ of points, lines, planes, $\cdots$, copoints of $L$ are its Whitney numbers of the second kind. Rota has conjectured that the sequence $\left\{W_{k}\right\}$ is unimodal: $w_{j} \geq \min \left\{W_{i}, W_{k}\right\}$ when $i \leq j \leq k$, a property known to hold for the partition lattices [8], [9], matroid designs [11], and all geometric lattices with eight or fewer points [3]. A related conjecture is $W_{r-k} \geq W_{k}$ when $k \leq r / 2$, which holds with equality for modular geometric lattices. The latter has been proved in the case $k=1[1],[6]$, and the equality $W_{r-1}=W_{1}$ shown to characterize modular geometric lattices [6]. Apparently little else is known in general about the sequence $\left\{W_{k}\right\}$, except that $W_{k} \geq W_{1}$ for $1 \leq k \leq r-1$, which follows easily from $W_{r-1} \geq W_{1} .(3)$ We prove here (Theorem 1) a lower bound for $W_{k}$ in terms of $k, r$, and the number $n=W_{1}$ of points of $L$, which improves the above bound substantially when $2 \leq k \leq r-2$. Our bound is attained only by the direct product of a modular plane and a free geometry when $2 \leq k \leq r-2$, and for these lattices equality holds for all $k$. In view of the representation of a finite lattice by its Hasse diagram, the term "slimmest" is accordingly a fitting description of these extremal lattices.

Presented to the Society, J anuary 25, 1973; received by the editors July 19, 1973. AMS (MOS) subject classifications (1970). Primary 05B35; Secondary 05A20.

(1) This research supported in part by NSF Grant GP-38951 (OSURF Project No. 3684 A1).

(2) This research supported in part by NSF Grant GP-28943 (OSURF Project No. 3228-A1).

(3) In [5] we prove $W_{1}+W_{2}+\cdots+W_{k} \leq W_{r-k}+\cdots+W_{r-2}+W_{r-1}$, for all $k$. 
The integers $w_{k}=\Sigma_{\mu}(0, x)$, the sum over all $x$ of rank $k$ in a geometric lattice $L$ with Möbius function $\mu$, are its Whitney numbers of the first kind. These are the coefficients in the characteristic (or chromatic) polynomial of $L$, of importance in the critical problem [4]. A well-known conjecture in graph theory asserts that the alternating sequence $\left\{w_{k}\right\}$ is unimodal in absolute value for graphic geometries, and empirical evidence suggests this may hold in general. An inequality for $w_{p}=\mu(0,1)$ in terms of the point-set-partition induced by a maximal chain in $L$ appears in [7]. We establish here (Theorem 2) a lower bound on $(-1)^{k} w_{k}$ in terms of $k, r$, and $n$, and show that equality holds when $k \geq 2$ only for the direct product of a line and a free geometry.

Our results are stated in $\$ 3$ following a brief section $(\$ 2)$ on preliminaries. In $\$ 4$ we verify that equality holds in Theorems 1 and 2 for the lattices described. The proofs of these theorems appear in $\$ 5$ and $\$ 6$, respectively.

2. Preliminaries, Definitions and results required in the sequel are summarized in this section. A detailed treatment of geometric lattices may be found in [2] or [4].

Let $L$ be a finite lattice. If $x \leq y$, the interval $[x, y]$ of $L$ is the sublattice $[x, y]=\{z \mid x \leq z \leq y\}$. An element $y$ covers $x$ iff $x<y$ and $x<z \leq y$ implies $z=y$, thus $[x, y]=\{x, y\}$. A point of $L$ is an element covering 0 , the minimum element of $L$. $\Lambda$ copoint of $L$ is an element covered by 1 , the maximum element of $L$. A chain of length $k$ in $L$ is a linearly-ordered subset $\left\{x_{i} \mid x_{0}<x_{1}<\cdots<x_{k}\right\}$ of $k+1$ elements. A maximal chain in $[x, y]$ is a chain $\left\{x_{i} \mid x_{0}<x_{1}<\ldots<x_{k}\right\}$ such that $x_{0}=x, x_{k}=y$, and $x_{i}$ covers $x_{i-1}, 1 \leq i \leq k, L$ satisfies the JordanDedekind chain condition iff all maximal chains in any interval $[x, y]$ are of equal length. In this case the rank $\rho(x)$ of $x \in L$ is the length of a maximal chain in $[0, x]$. The rank of $L$ is the rank of its unit element 1 .

A finite lattice $L$ is geometric when $y$ covers $x$ is equivalent to $y=x \vee p$ for some point $p \notin x$. Equivalently, the elements covering an element $x<1$ partition the set of points $\measuredangle x$. The Jordan-Dedekind chain condition holds in a geometric lattice, and its rank function satisfies the semimodular inequality $\rho(x \vee y)+\rho(x \wedge y) \leq \rho(x)+\rho(y)$. L is modular if equality holds for all $x, y$. If $L$ is geometric of rank $r$, elements of rank $1,2,3, r-1$ are points, lines, planes, copoints, respectively. Every interval of a geometric lattice is geometric, and direct products of (modular) geometric lattices are (modular) geometric lattices.

Geometric lattices are the order-theoretic counterparts of combinatorial geometries [4], or matroids, the elements of the lattice representing the closed subsets of points of the geometry, ordered by inclusion. We shall employ geometrical language where convenient in arguments below. 
A separator of a geometric lattice $L$ is an element $x \neq 0,1$ such that $L \simeq$ $[0, x] \times[x, 1]$. If $x$ is a separator, then so is $y=V\{p \mid p$ a point $\not x\}$, and the mapping $z \mapsto z \vee x$ is an isomorphism between $[0, y]$ and $[x, 1] . L$ is connected if it has no separators. An isthmus is a separator which is a point of $L$. Then $p$ is an isthmus of $L$ iff there is a copoint $c$ such that $q \leq c$ for every point $q \neq p$.

The truncation of a geometric lattice $L$ of rank $r$ is the subset $\{x \in L \mid \rho(x) \neq$ $r-1\}$ in the induced order, a geometric lattice of rank $r-1$. By a sequence of $r-k$ truncations $L$ may be reduced to a geometric lattice $L^{\prime}$ of rank $k$ whose copoints are the elements of rank $k-1$ in $L$. We call $L^{\prime}$ the truncation of $L$ to rank $k_{\text {. }}$

The free geometry (boolean algebra) with $j$ points is the geometric lattice (of rank $j$ ) in which every point is an isthmus, and is isomorphic to the lattice of all subsets of its point set under the inclusion order. $\Lambda$-point line is a geomettic lattice of rank two with $j$ points. $\Lambda j$-point projective plane is a connected, modular geometric lattice of rank three with $j$ points. Each of these three types of lattices is modular, hence so are direct products of them.

The Möbius function [10] of a finite lattice $L$ is the function $\mu: L \times L \rightarrow Z$ defined recursively by $\mu(x, y)=0$ if $x \not y, \mu(x, y)=1$ if $x=y$, and $\mu(x, y)=$ $-\Sigma\{\mu(x, z) \mid x \leq z<y\}$ if $x<y$.

3. Main results, Let $L$ be a finite geometric lattice of rank $r$ with $n$ points and rank function $\rho$. The Whitney numbers of $L$, of the second kind, are the integers

$$
W_{k}=|\{x \in L \mid \rho(x)=k\}|, \quad 0 \leq k \leq r .
$$

Thus $W_{0}=W_{r}=1$ and $W_{1}=n$ by definition. Basterfield and Kelly [1] and Greene [6] proved the inequality

$$
W_{k} \geq n, \quad 1 \leq k \leq r-1 .
$$

Greene showed further that equality holds in (3.2) for some $k, 2 \leq k \leq r-1$, iff $k=r-1$ and $L$ is modular. If $1 \leq k<r-1,(3.2)$ follows immediately from

$$
w_{r-1} \geq n,
$$

on application of (3.3) to the truncation of $L$ to rank $k+1$. Inequality (3.2) is strengthened substantially when $2 \leq k \leq r-2$ by

Theorem 1. Let $L$ be a finite geometric lattice of rank $r$ with $n$ points. Then

$$
W_{k} \geq\left(\begin{array}{l}
r-2 \\
k-1
\end{array}\right)(n-r)+\left(\begin{array}{l}
r \\
k
\end{array}\right), \quad 0 \leq k \leq r
$$


When $r \geq 4$, equality holds in (3.4) for some $k, 2 \leq k \leq r-2$, iff $L$ is (isomorphic to) the direct product of a modular plane and a free geometry.

By Greene's result, the latter conclusion is valid also when equality holds in (3.4) for $k=2, r=3$, in which case the free geometry is trivial (rank zero).

The extremal lattices may be described in greater detail. A modular plane is either projective, or if not connected, the direct product of a line and a one-point free geometry. Denote by $F_{j}, Q_{j}, P_{j}$ a $j$-point free geometry, line, and (arbitrary) projective plane, respectively, and let

$$
\begin{aligned}
& Q(i, j)=Q_{i} \times F_{j}, \\
& P(i, j)=P_{i} \times F_{j} .
\end{aligned}
$$

Then the conclusion when equality holds in (3.4) may be stated:

$$
L \cong Q(n-r+2, r-2) \text { or } L \cong P(n-r+3, r-3)
$$

The Whitney numbers of the first kind are the integers

$$
w_{k}=\sum_{\rho(x)=k} \mu(0, x), \quad 0 \leq k \leq r,
$$

$\mu$ being the Möbius function of $L$. Since $\mu(x, y)$ is nonzero when $x \leq y$, with sign $(-1)^{\rho(y)-\rho(x)}[10], w_{k}$ is nonzero with sign $(-1)^{k}$. Thus

$$
w_{k}^{+}=(-1)^{k} w_{k}
$$

is positive. Trivially, $w_{0}^{+}=1$ and $w_{1}^{+}=n$.

Theorem 2. Let $L$ be a finite geometric lattice of rank $r$ with $n$ points. Then

$$
w_{k}^{+} \geq\left(\begin{array}{c}
r-1 \\
k-1
\end{array}\right)(n-r)+\left(\begin{array}{l}
r \\
k
\end{array}\right), \quad 0 \leq k \leq r .
$$

Equality holds in (3.9) for some $k, 2 \leq k \leq r$, iff $L$ is isomorphic to the direct product of a line and a free geometry.

Thus $L \cong Q(n-r+2, r-2)$ when equality holds.

4. The extremal lattices. In this section we verify that equality holds in (3.4) for $Q(n-r+2, r-2)$ and $P(n-r+3, r-3)$ and in (3.9) for $Q(n-r+2, r-2)$. In the proof of Theorem $2(\$ 6)$, we shall require the fact that (3.9) is a strict inequality when $k \geq 2$ for $P(n-r+3, r-3)$, a result most conveniently established at this point. 
In computing the Whitney numbers of a direct product, it is useful to consider the polynomials

$$
\begin{aligned}
& \phi(\lambda)=\sum_{x \in L} \lambda^{r-\rho(x)}, \\
& \chi(\lambda)=\sum_{x \in L} \mu(0, x) \lambda^{r-\rho(x),}
\end{aligned}
$$

where $r$ is the rank of $L$. Thus the coefficient of $\lambda^{r-k}$ is $W_{k}$ in (4.1) and $w_{k}$ in (4.2). The latter is the characteristic polynomial of $L$.

For a direct product $L=L_{1} \times L_{2}$ of geometric lattices, $\rho((x, y))=\rho_{1}(x)+$ $\rho_{2}(y)$ and $\mu((0,0),(x, y))=\mu_{1}(0, x) \mu_{2}(0, y)[10]$; thus

$$
\phi(\lambda)=\phi_{1}(\lambda) \phi_{2}(\lambda)
$$

$$
\chi(\lambda)=\chi_{1}(\lambda) \chi_{2}(\lambda)
$$

The polynomials $\phi(\lambda), \chi(\lambda)$ are well known for a free geometry, line, and projective plane, and are given below in Table 1 . Since the existence of $P_{j}$ implies $j=s^{2}+s+1$ for some integer $s \geq 2$, we make this substitution where convenient.

\begin{tabular}{|c|c|c|}
\hline & $\phi(\lambda)$ & $\chi(\lambda)$ \\
\hline$F_{j}$ & $\sum_{i=0}^{j}\left(\begin{array}{l}j \\
i\end{array}\right) \lambda^{j-i}$ & $\sum_{i=0}^{j}(-1)^{i}\left(\begin{array}{l}j \\
i\end{array}\right) \lambda^{j-i}$ \\
\hline$Q_{j}$ & $\lambda^{2}+j \lambda+1$ & $\lambda^{2}-j \lambda+(j-1)$ \\
\hline$P_{j}$ & $\lambda^{3}+j \lambda^{2}+j \lambda+1$ & $\lambda^{3}-\left(s^{2}+s+1\right) \lambda^{2}$ \\
& & $+s\left(s^{2}+s+1\right) \lambda-s^{3}$, \\
& & $j=s^{2}+s+1$ \\
\hline
\end{tabular}

Table 1

From (4.3) and Table 1 we obtain the $\phi(\lambda)$-coefficient of $\lambda^{r-k}$ for $Q(n-r+2, r-2), P(n-r+3, r-3)$, respectively, as 


$$
\begin{gathered}
\left(\begin{array}{c}
r-2 \\
k
\end{array}\right)+\left(\begin{array}{c}
r-2 \\
k-1
\end{array}\right)(n-r+2)+\left(\begin{array}{c}
r-2 \\
k-2
\end{array}\right), \\
\left(\begin{array}{c}
r-3 \\
k
\end{array}\right)+\left(\begin{array}{c}
r-3 \\
k-1
\end{array}\right)(n-r+3)+\left(\begin{array}{c}
r-3 \\
k-2
\end{array}\right)(n-r+3)+\left(\begin{array}{c}
r-3 \\
k-3
\end{array}\right) .
\end{gathered}
$$

Using Pascal's identity, both (4.4) and (4.5) reduce to the right-hand side of (3.4).

From (4.4) and Table 1 we obtain the $\chi(\lambda)$-coefficient of $(-1)^{k} \lambda^{r-k}$ for $Q(n-r+2, r-2)$ as

$$
\left(\begin{array}{c}
r-2 \\
k
\end{array}\right)+(n-r+2)\left(\begin{array}{c}
r-2 \\
k-1
\end{array}\right)+(n-r+1)\left(\begin{array}{c}
r-2 \\
k-2
\end{array}\right)
$$

which simplifies to the right-hand side of (3.9), and for $P(n-r+3, r-3)$ as

$$
\left(\begin{array}{c}
r-3 \\
k
\end{array}\right)+\left(s^{2}+s+1\right)\left(\begin{array}{c}
r-3 \\
k-1
\end{array}\right)+s\left(s^{2}+s+1\right)\left(\begin{array}{l}
r-3 \\
k-2
\end{array}\right)+s^{3}\left(\begin{array}{c}
r-3 \\
k-3
\end{array}\right)
$$

Setting $n-r+3=s^{2}+s+1$ and using Pascal's identity, we can rewrite (4.6) as

$$
\left(\begin{array}{c}
r-3 \\
k
\end{array}\right)+\left(s^{2}+s+1\right)\left(\begin{array}{c}
r-3 \\
k-1
\end{array}\right)+2\left(s^{2}+s-1 / 2\right)\left(\begin{array}{l}
r-3 \\
k-2
\end{array}\right)+\left(s^{2}+s-1\right)\left(\begin{array}{l}
r-3 \\
k-3
\end{array}\right)
$$

The first two terms in (4.7) and (4.8) are equal, but if $2 \leq k \leq r$, at least one of $\left(\begin{array}{l}r-3 \\ k-2\end{array}\right),\left(\begin{array}{c}r-3 \\ k-3\end{array}\right)$ is positive. Since $s \geq 2, s\left(s^{2}+s+1\right)>2\left(s^{2}+s-1 / 2\right)$ and $s^{3}>s^{2}+s-1$, hence (4.7) is strictly greater than (4.8) when $2 \leq k \leq r$.

5. Proof of Theorem 1. We proceed now to the proof of inequality (3.4). The following notation will be used, for an interval $[u, v]$ of $L$.

$$
\begin{array}{ll}
A_{j}(u, v)=\{x \in[u, v] \mid \rho(x)=j\}, & a_{j}(u, v)=\left|A_{j}(u, v)\right| . \\
B_{j}(u, v)=\{x \notin[u, v] \mid \rho(x)=j\}, & \beta_{j}(u, v)=\left|B_{j}(u, v)\right| .
\end{array}
$$

Thus $W_{j}=a_{j}(u, v)+\beta_{j}(u, v)$. Since $A_{j}(u, v)$ is the set of elements of rank $j-\rho(u)$ in the interval $[u, v]$, we have

$$
W_{j-\rho(u)}([u, v])=a_{j}(u, v)
$$

We shall require the following lemma. A proof is given in our related paper $[5$, Corollary to Theorem 4].

Lemma. For any point $p$ of $L$,

$$
\alpha_{2}(p, 1)+\beta_{r-1}(p, 1) \geq \alpha_{1}(0,1) .
$$


The proof of (3.4) will be by induction on the sum $r+k$. When $r+k \leq 5$, the only nontrivial case is $r=3, k=2$, where (3.4) follows from (5.2) (or from (3.3)). As the inductive hypothesis we assume that if $L^{\prime}$ is a geometric lattice of rank $r^{\prime}$ with $n^{\prime}$ points, then (3.4) holds for all $k^{\prime}$ such that $r^{\prime}+k^{\prime}<r+k$, where $r+$ $k \geq 6$. By (3.3) we may assume $2 \leq k \leq r-2$, so $r \geq 4$.

Fix a point $p$ of $L$. Then

$$
W_{k}=a_{k}(p, 1)+\beta_{k}(p, 1) .
$$

Let $l=a_{2}(p, 1)=W_{1}([p, 1])$. The interval $[p, 1]$ is of rank $r-1$, so by $(5.1)$ and the inductive hypothesis,

$$
a_{k}(p, 1) \geq\left(\begin{array}{l}
r-3 \\
k-2
\end{array}\right)(l-r+1)+\left(\begin{array}{l}
r-1 \\
k-1
\end{array}\right)
$$

To obtain a lower bound on $\beta_{k}(p, 1)$, we first observe that $y \in A_{k+1}(p, 1)$ iff $y=$ $x \vee p$ for some $x \in B_{k}(p, 1)$. The mapping $x \mapsto x \vee p$ is thus a surjection $B_{k}(p, 1) \rightarrow A_{k+1}(p, 1)$, and so partitions $B_{k}(p, 1)$ into inverse images of elements of $A_{k+1}(p, 1)$. The inverse image of $y \in A_{k+1}(p, 1)$ is the subset $A_{k}(0, y)-A_{k}(p, y)$ of $B_{k}(p, 1)$, of cardinality $a_{k}(0, y)-a_{k}(p, y)$. By (5.2), $a_{k}(0, y)-a_{k}(p, y) \geq a_{1}(0, y)-a_{2}(p, y)$. Thus

$$
\beta_{k}(p, 1) \geq \sum_{y \in A_{k+1}(p, 1)} a_{1}(0, y)-\sum_{y \in A_{k+1}(p, 1)} a_{2}(p, y) .
$$

The two sums in (5.5) may be written as follows on interchanging the order of summation in each.

$$
\begin{gathered}
\sum_{y \in A_{k+1}(p, 1)} a_{1}(0, y)=a_{k+1}(p, 1)+\sum_{q \in B_{1}(p, 1)} a_{k+1}(p \vee q, 1) \\
=a_{k+1}(p, 1)+\sum_{a \in A_{2}(p, 1)}\left(a_{1}(0, a)-1\right) a_{k+1}(a, 1) . \\
\sum_{y \in A_{k+1}(p, 1)} a_{2}(p, y)=\sum_{a \in A_{2}(p, 1)} a_{k+1}(a, 1) .
\end{gathered}
$$

Substituting (5.6) and (5.7) into (5.5) gives

$$
\beta_{k}(p, 1) \geq a_{k+1}(p, 1)+\sum_{a \in A_{2}(p, 1)}\left(a_{1}(0, a)-2\right) a_{k+1}(a, 1)
$$

Writing $a_{k+1}(a, 1)=a_{k+1}(a, 1)-\left(\begin{array}{c}r-2 \\ k-1\end{array}\right)+\left(\begin{array}{c}r-2 \\ k-1\end{array}\right)$ in (5.8), and noting that 
$\Sigma_{a \in A_{2}(p, 1)}\left(a_{1}(0, a)-2\right)=n-1-l$, we obtain

$$
\beta_{k}(p, 1) \geq a_{k+1}(p, 1)+\left(\begin{array}{c}
r-2 \\
k-1
\end{array}\right)(n-1-l)+C_{p},
$$

where

$$
C_{p}=\sum_{a \in A_{2}(p, 1)}\left(a_{1}(0, a)-2\right)\left(\alpha_{k+1}(a, 1)-\left(\begin{array}{c}
r-2 \\
k-1
\end{array}\right)\right) \geq 0,
$$

since $a_{1}(0, a)=W_{1}([0, a]) \geq 2$ and $a_{k+1}(a, 1)=W_{k-1}([a, 1]) \geq(r-2)$. The interval $[a, 1]$ is of rank $r-2$, and $2 \leq k \leq r-2$ implies $1 \leq k-1 \leq(r-2)-1$, so $W_{k-1}([a, 1])=\left(\begin{array}{c}r-2 \\ k-1\end{array}\right)$ iff $[a, 1] \simeq F_{r-2^{\circ}}$ Thus equality holds in (5.10) iff for every line $a$ on $p$, either $[0, a] \simeq F_{2}$ or $[a, 1] \simeq F_{r-2^{*}}$.

The interval $[p, 1]$ is of rank $r-1$, so by (5.1) and the inductive hypothesis

$$
a_{k+1}(p, 1) \geq\left(\begin{array}{c}
r-3 \\
k-1
\end{array}\right)(l-r+1)+\left(\begin{array}{c}
r-1 \\
k
\end{array}\right) \text {. }
$$

We obtain finally, from (5.3), (5.4), (5.9), (5.10), and (5.11),

$$
\begin{aligned}
W_{k} \geq & \left(\begin{array}{l}
r-3 \\
k-2
\end{array}\right)(l-r+1)+\left(\begin{array}{l}
r-1 \\
k-1
\end{array}\right)+\left(\begin{array}{l}
r-3 \\
k-1
\end{array}\right)(l-r+1) \\
& +\left(\begin{array}{c}
r-1 \\
k
\end{array}\right)+\left(\begin{array}{c}
r-2 \\
k-1
\end{array}\right)(n-1-l) \\
& =\left(\begin{array}{l}
r-2 \\
k-1
\end{array}\right)(n-r)+\left(\begin{array}{l}
r \\
k
\end{array}\right) .
\end{aligned}
$$

Suppose now that $2 \leq k \leq r-2$ and equality holds in (3.4). Then equality holds in (5.4) and (5.11) for every point $p$ of $L$, where $l=a_{2}(p, 1)$, and equality holds in (5.10) for every line $a$ of $L$. To prove that $L \cong Q(n-r+2, r-2)$ or $L \simeq P(n-r+3, r-3)$, we again argue by induction on the sum $r+k$. Thus assume that if $L^{\prime}$ is a geometric lattice of rank $r^{\prime}$ with $n^{\prime}$ points, and equality holds in (3.4) for some $k^{\prime}, 2 \leq k^{\prime} \leq r^{\prime}-2$, then $L^{\prime} \cong Q\left(n^{\prime}-r^{\prime}+2, r^{\prime}-2\right)$ or $L^{\prime} \simeq Q\left(n^{\prime}-r^{\prime}+2, r^{\prime}-3\right)$, whenever $r^{\prime}+k^{\prime}<r+k$. The initial case is $r+$ $k=6$, when $r=4, k=2$. As the proof for this case is similar to the inductive step, it will be convenient to postpone it. Thus we assume $r+k \geq 7$, so $r \geq 5$. Then at least one of the pairs $\left(r^{\prime}, k^{\prime}\right)=(r-1, k-1),(r-1, k)$ satisfies $2 \leq$ $k^{\prime} \leq r^{\prime}-2$, so by the inductive hypothesis, for every point $p$ of $L$, either 


$$
[p, 1] \cong Q(l-r+3, r-3)
$$

or

$$
[p, 1] \cong P(l-r+4, r-4),
$$

where $l=a_{2}(p, 1)$ is the number of lines of $L$ on $p$. Also, from equality in (5.10) we have for every line $a$ of $L$, either $[0, a] \simeq F_{2}$ or $[a, 1] \simeq F_{r-2^{\circ}}$

Suppose first that every line of $L$ has two points $\left([0, a] \cong F_{2}\right)$. Let $p$ be a point of $L$. Since $r \geq 5,(5.12)-(5.13)$ imply that $[p, 1]$ has an isthmus $b$. Then in $L, b$ is the only line on $p$ not on some copoint $c$ on $p$. The second point $q$ on $a$ is thus the only point of $L$ not on $c$, so $q$ is an isthmus of $L$. The number of lines on $q$ is $n-1$, so by (5.12)-(5.13),

$$
L \cong[0, c] \times[0, q] \cong[p, 1] \times F_{1} \cong\left\{\begin{array}{l}
Q(n-r+2, r-2) \\
\text { or } \\
P(n-r+3, r-3)
\end{array}\right.
$$

Suppose now that there is a line $a$ of $L$ with at least three points. Then $[a, 1] \cong F_{r-2^{*}}$. Let $u_{1}, u_{2}, \cdots, u_{r-2}$ be the planes on $a$. The join of any set of $j$ of these is an element of rank $j+2$ in $L$. Let $p$ be a point of $a$ and suppose first that (5.13) holds. Then there is an element $x$ of rank four in $L$ such that $x>p$ and $[p, x]$ is a projective plane. There are then two points $p_{1}, p_{2}$ of $x$ on different planes through $a$, say $u_{1}, u_{2}$, respectively. The plane $p \vee p_{1} \vee p_{2}$ of $x$ intersects $u_{i}, 3 \leq i \leq r-2$, only in $p$, as otherwise $u_{1} \vee u_{2} \vee u_{i}$ is of rank four. But then $x$ contains only two lines $p \vee p_{1}, p \vee p_{2}$ on $p$, contradicting that $[p, x]$ is a projective plane, hence has no two-point lines. Thus we may assume that (5.12) holds for every point $p$ on $a$, so at most one of the planes on $a$ contains more than one line on $p$ other than $a$. Suppose $u_{1}, u_{2}$ each contain two points off $a$, say $p_{1}, q_{1}$ in $u_{1}$ and $p_{2}, q_{2}$ in $u_{2}$. Since $a$ has at least three points, there is a point $p$ of $a$ not on either of the lines $p_{1} \vee q_{1}, p_{2} \vee q_{2}$. We then have three lines $a, p \vee p_{i}, p \vee q_{i}$ on $p$ in $u_{i}, i=1,2$, a contradiction. Thus $u_{1}$, say, has only a single point $q$ off $a$. Then $q$ is the only point of $L$ off the copoint $c=u_{2} \vee \ldots \vee u_{r-2}$, hence is an isthmus of $L$. The number of lines on $q$ is $l=n-1$, and the argument preceding (5.14) can be repeated.

It remains only to verify the result for the case $r=4, k=2$. From equality in (5.11) we have, by Greene's result, that $[p, 1]$ is a modular plane for every point $p$ of $L$. If there is a nontrivial line $a$ in $L$, the argument above gives an isthmus $q$ on one of the two planes through $a$, and we are finished as before. If every line has two points, then by equality in (3.4) we have $W_{2}=\left(\begin{array}{l}n \\ 2\end{array}\right)=2 n-2$, which implies $n=r=4$, so $L \cong F_{4} \cong Q(2,2)$. 
6. Proof of Theorem 2. We consider first the case $k=r$ in Theorem 2. Let $\mu^{+}(0, x)=(-1)^{\rho(x)} \mu(0, x)$, a positive integer. If $p$ is a point of $L$, we have by Weisner's the orem [10],

$$
\mu^{+}(0,1)=\sum \mu^{+}(0, c)
$$

where the sum is over all copoints $c$ of $L$ such that $c \ngtr p$.

Proposition 1. If $L$ is a geometric lattice of rank $r$ with $n$ points, then

$$
\mu^{+}(0,1) \geq n-r+1
$$

with equality iff $L \cong Q(n-r+2, r-2)$.

Proof. The proof is by induction on $r$. Equality holds in (6.2) for $r=2$, so assume inductively that (6.2) holds for $r^{\prime}<r$, where $r \geq 3$. If $c$ is a copoint of $L$, let $\alpha(c)$ denote the number of points in $[0, c]$. By the inductive hypothesis,

$$
\mu^{+}(0, c) \geq a(c)-r+2 \text {. }
$$

Summing (6.2) over all points $p$ of $L$, and using (6.3), we obtain

$$
n \mu^{+}(0,1) \geq \sum_{c \text { copoint }}(n-\alpha(c))(a(c)-r+2)
$$

But for any copoint $c, r-1 \leq a(c) \leq n-1$, so

$$
\begin{aligned}
(n-\alpha(c))(\alpha(c)-r+2) & =(n-1-\alpha(c))(\alpha(c)-(r-1))+n-r+1 \\
& \geq n-r+1,
\end{aligned}
$$

with equality iff $a(c)=r-1$ or $n-1$. Thus from (6.4), (6.5), and (3.3)

$$
\mu^{+}(0,1) \geq W_{r-1}(n-r+1) / n \geq n-r+1 \text {. }
$$

To show that equality in (6.2) implies $L \simeq Q(n-r+2, r-2)$, we again argue by induction on $r$. The result is trivially true if $r=2$, so assume $r \geq 3$, and that equality holds in (6.2). By (6.6), $W_{r-1}=n$, and from (6.5) every copoint of $L$ has either $r-1$ or $n-1$ points. If all copoints have $r-1$ points, then either $L \simeq F_{r} \simeq Q(2, r-2)$, or else $L$ is the truncation to rank $r$ of a free geometry $F_{n}, n>r$. But in the latter case, $W_{r-1}=\left(\begin{array}{c}n \\ -_{1}\end{array}\right)>n$. If some copoint $c$ contains $n-1$ points, the point $p$ not on $c$ is an isthmus of $L$. Since equality must hold in (6.3) for every copoint, $[0, c] \cong Q(n-r+2, r-3)$. Then $L \cong$ $[0, c] \times[0, p] \simeq Q(n-r+2, r-2)$, and the lemma is proved.

Let $A_{i}=\{x \in L \mid \rho(x)=i\}$ and for $x \in L$, let $\alpha(x)$ be the number of points in $[0, x]$. Define $m_{k}^{+}=\Sigma_{x \in A_{k}} a(x) \mu^{+}(0, x)$. We then have 
Proposition 2. Let $L$ be a geometric lattice of rank $r$ with $n$ points. Then

$$
m_{k}^{+} \geq(n-r+1) W_{k-1}+(r-k) w_{k-1}^{+}, \quad 1 \leq k \leq r
$$

Proof. The case $k=1$ is trivial, and the first inequality in $(6.6)$ is the case $k=r$, so assume $2 \leq k \leq r-1$. Then by applying $(6.1)$ to the interval $[0, x]$ below, we obtain

$$
\begin{aligned}
m_{k}^{+} & =\sum_{x \in A_{k}} \sum_{p \in A_{1}, x>p} \mu^{+}(0, x)=\sum_{p \in A_{1}} \sum_{x \in A_{k}, x>p} \mu^{+}(0, x) \\
& =\sum_{p \in A_{1}} \sum_{x \in A_{k^{\prime}} x>p}\left(\sum_{y \in A_{k-1}, p \leq y<x} \mu^{+}(0, y)\right) \\
& =\sum_{y \in A_{k-1}} \mu^{+}(0, y) \sum_{p \in A_{1}, p \leq y \leq x \in A_{k}, x=p \vee y} 1 \\
& =\sum_{y \in A_{k-1}} \mu^{+}(0, y)(n-a(y)) \\
& =\sum_{y \in A_{k-1}} \mu^{+}(0, y)(n-a(y)-r+k)+(r-k) \sum_{y \in A_{k-1}} \mu^{+}(0, y) .
\end{aligned}
$$

We next apply (6.2) to the interval $[0, y]$.

$$
\begin{aligned}
m_{k}^{+} & \geq \sum_{y \in A_{k-1}}(a(y)-k+2)(n-a(y)-r+k)+(r-k) w_{k}^{+} \\
& \geq(n-r+1) W_{k-1}+(r-k) w_{k}^{+},
\end{aligned}
$$

the last inequality following since $k-1 \leq a(y) \leq n-r+k-1$ for $y$ of rank $k-1$, so

$$
\begin{aligned}
(a(y)- & k+2)(n-a(y)-r+k) \\
& =(a(y)-k+1)(n-r+k-1-a(y))+(n-r+1) \\
& \geq n-r+1 .
\end{aligned}
$$

We consider now inequality (3.9), arguing by induction on $k_{0}$. The case $k=1$ is trivial, and by (6.2) we may assume $k<r$, so let $2 \leq k \leq r-1$ and suppose (3.9) holds for all $k^{\prime}<k$.

Let $p$ be a point of $L$. Applying $(6.1)$ to $[0, y]$, for $y \in A_{k+1}, y>p$, we obtain 


$$
\begin{aligned}
w_{k}^{+} & =\sum_{x \in A_{k}} \mu^{+}(0, x)=\sum_{x \in A_{k^{\prime}} x>p} \mu^{+}(0, x)+\sum_{x \in A_{k^{\prime}} x \neq p} \mu^{+}(0, x) \\
& =\sum_{x \in A_{k^{\prime}} x>p} \mu^{+}(0, x)+\sum_{y \in A_{k+1}, y>p} \mu^{+}(0, y) .
\end{aligned}
$$

Summing over all points $p$, and using (6.7),

$$
\begin{aligned}
n w_{k}^{+} & =\sum_{x \in A_{k}} a(x) \mu^{+}(0, x)+\sum_{y \in A_{k+1}} a(y) \mu^{+}(0, y)=m_{k}^{+}+m_{k+1}^{+} \\
& \geq(n-r+1)\left(W_{k-1}+W_{k}\right)+(r-k) w_{k-1}^{+}+(r-k-1) w_{k}^{+} .
\end{aligned}
$$

We now apply (3.4) and the inductive hypothesis, obtaining

$$
\begin{aligned}
(n-r+1+k) w_{k}^{+} \geq(n-r+1)\left(W_{k-1}+W_{k}\right)+(r-k) w_{k-1}^{+} \\
\geq(n-r+1)\left\{\left(\begin{array}{l}
r-2 \\
k-2
\end{array}\right)(n-r)+\left(\begin{array}{c}
r \\
k-1
\end{array}\right)+\left(\begin{array}{l}
r-2 \\
k-1
\end{array}\right)(n-r)+\left(\begin{array}{l}
r \\
k
\end{array}\right)\right\} \\
+(r-k)\left\{\left(\begin{array}{l}
r-1 \\
k-2
\end{array}\right)(n-r)+\left(\begin{array}{c}
r \\
k-1
\end{array}\right)\right\} \\
=(n-r+1)\left\{\left(\begin{array}{l}
r-1 \\
k-1
\end{array}\right)(n-r)+\left(\begin{array}{l}
r \\
k
\end{array}\right)\right\}+k\left\{\left(\begin{array}{l}
r-1 \\
k-1
\end{array}\right)(n-r)+\left(\begin{array}{l}
r \\
k
\end{array}\right)\right\}
\end{aligned}
$$

after simplification. Thus

$$
(n-r+1+k) w_{k}^{+} \geq(n-r+1+k)\left\{\left(\begin{array}{c}
r-1 \\
k-1
\end{array}\right)(n-r)+\left(\begin{array}{l}
r \\
k
\end{array}\right)\right\}
$$

so (3.9) follows.

To complete the proof of Theorem 2, assume equality holds in (3.9) for some $k, 2 \leq k \leq r$. If $k=r$, the result follows from Proposition 1 , so suppose $2 \leq k \leq$ $r-1$. The proof is by induction on $r$. Consider first the case $r=3, k=2$. From (6.8), equality in (3.9) implies $W_{2}=n$, so $L$ is a modular plane, and therefore $L \cong Q(n-1,1)$ or $L \cong P(n, 0)$. But the latter is impossible $(\$ 4)$, hence $L \cong$ $Q(n-1,1)$.

Assume inductively that the result holds for $r^{\prime}<r$, where $r \geq 4$. Then equality in (3.9) implies equality in (6.8), so $W_{k}, W_{k-1}$ attain the lower bound in (3.4). At least one of $k-1, k$ satisfy $2 \leq k^{\prime} \leq r-2$, so by Theorem 1 , either $L \cong Q(n-r+2, r-2)$ or $L \cong P(n-r+3, r-3)$. But $k \geq 2$, so again the latter is impossible, and the proof is complete. 


\section{REFERENCES}

1. J. G. Basterfield and L. M. Kelly, A characterization of sets of $n$ points which determine $n$ hyperplanes, Proc. Cambridge Philos. Soc. 64 (1968), 585-588. MR 38 $\# 2040$.

2. G. Birkhoff, Lattice theory, 3rd ed., Amer. Math. Soc. Colloq. Publ., vol. 25, Amer. Marh. Soc., Providence, R. I., 1967. MR 37 \#2638.

3. J. E. Bl ack bum, H. H. Crapo and D. A. Higgs, A catalogue of combinatorial geom. etries, University of Waterloo, Waterloo, Ontario, 1969.

4. H. H. Crapo and G.-C. Rota, On the foundations of combinatorial theory: Combinatorial geometries, Preliminary edition, M. I. T. Press, Cambridge, Mass., 1970. MR 45 \#74.

5. T. A. Dowling and R. M. Wilson, Whitney number inequalities for geometric lattices, Proc. Amer. Math. Soc. (to appear).

6. C. Greene, A rank inequality for finite geometric lattices, J. Combinatorial Theory 9 (1970), 357-364. MR $42 \# 1727$.

7. - An inequality for the Möbius function of a geometric lattice, Proc. Conference on Möbius Algebras (H. Crapo and G. Roulet, ed.), University of Waterloo, 1971.

8. L. H. Harper, Stirling behavior is asymptotically normal, Ann. Math. Statist. 38 (1967), 410-414. MR $35 \# 2312$.

9. E. H. Lieb, Concavity properties and a generating function for Stirling numbers, J. Combinatorial Theory 5 (1968), 203-206. MR 37 \#6195.

10. G.-C. Rota, On the foundations of combinatorial theory. I. Theory of Möbius functions, Z. Wahrscheinlichkeitstheorie und Verw. Gebiete 2(1964), 340-368. MR 30 \#4688.

11. P. Young, U. S. R. Murty and J. Edmonds, Equicardinal matroids and matroiddesigns, Proc. Second Chapel Hill Conf. on Combinatorial Mathematics and its Applications, Univ. of North Carolina, Chapel Hill, N. C., 1970, pp. 498-542. MR 42 \#1685.

DEPARTMENT OF MATHEMATICS, OHIO STATE UNIVERSITY, COLUMBUS, OHIO 43210 